\title{
Gene overexpression screen for chromosome instability in yeast primarily identifies cell cycle progression genes
}

\author{
Hanna Tutaj ${ }^{1}$ Elzbieta Pogoda ${ }^{1} \cdot$ Katarzyna Tomala $^{1} \cdot$ Ryszard Korona $^{1}$ \\ Received: 8 June 2018 / Revised: 11 September 2018 / Accepted: 12 September 2018 / Published online: 22 September 2018 \\ (C) The Author(s) 2018
}

\begin{abstract}
Loss of heterozygosity (LOH) in a vegetatively growing diploid cell signals irregularity of mitosis. Therefore, assays of LOH serve to discover pathways critical for proper replication and segregation of chromosomes. We screened for enhanced $\mathrm{LOH}$ in a whole-genome collection of diploid yeast strains in which a single gene was strongly overexpressed. We found 39 overexpression strains with substantially increased LOH caused either by recombination or by chromosome instability. Most of them, 32 in total, belonged to the category of "cell division", a broadly defined biological process. Of those, only one, TOP3, coded for an enzyme that uses DNA as a substrate. The rest related to establishment and maintenance of cell polarity, chromosome segregation, and cell cycle checkpoints. Former studies, in which gene deletions were used, showed that an absence of a protein participating in the DNA processing machinery is a potent stimulator of genome instability. As our results suggest, overexpression of such proteins is not comparably damaging as the absence of them. It may mean that the harmful effect of overexpression is more likely to occur in more complex and multistage processes, such as chromosome segregation. We also report a side finding, resulting from the fact that we worked with the yeast strains bearing a 2-micron plasmid. We noted that intense transcription from such a plasmid led to an enhanced rate of an entire chromosome loss (as opposed to $\mathrm{LOH}$ produced by recombination). This observation may support models linking segregation of 2-micron plasmids to segregation of chromosomes.
\end{abstract}

Keywords Saccharomyces cerevisiae $\cdot$ Chromosome loss $\cdot$ Chromosome recombination $\cdot$ 2-Micron plasmid $\cdot$ Mitosis

\section{Introduction}

Events of unequal distribution of DNA to daughter cells upon mitosis can be collectively described as chromosomal instability (CIN). One approach to study CIN is to detect and compare the existing aberrant genomes. However, chromosomal mutations are typically non-neutral to fitness. Some can be beneficial and fixed by natural selection (Comai 2005; Harari et al. 2018; Otto and Whitton 2000; Torres et al. 2008). More frequently, they are deleterious and,

Communicated by M. Kupiec.

Electronic supplementary material The online version of this article (https://doi.org/10.1007/s00294-018-0885-x) contains supplementary material, which is available to authorized users.

Ryszard Korona

ryszard.korona@uj.edu.pl

1 Institute of Environmental Sciences, Jagiellonian University, Gronostajowa 7, 30-387 Kraków, Poland therefore, eliminated either immediately or quickly enough to pass overlooked (Sheltzer and Amon 2011). The deleterious changes known from genetic counselling constitute probably only a small and non-random sample of all such events (Duijf and Benezra 2013; Hui and Bianchi 2013). It is, therefore, advisable to do systematic genetic screens of possibly nascent CINs, even though they usually involve specifically engineered and thus rather narrowly focused detection systems (Duffy et al. 2016; Dunham and Fowler 2013).

Genome-wide screens for factors affecting a trait of question are routinely done with a handful of model species of which Saccharomyces cerevisiae is outstanding both in terms of opportunities offered and results already obtained (Boone 2014; Giaever and Nislow 2014; Scherens and Goffeau 2004). The previous systematic screens for genes affecting CIN in this species can be divided according to two criteria. One is the way in which activity of genes potentially linked to CIN was affected: either completely absent (deletion studies) or just modified (expression studies). The other 
is the kind of sensor of genetic change: either loss of an additional chromosome fragment (within a haploid or diploid cell) or loss of heterozygosity ( $\mathrm{LOH}$ ) at a locus residing on a regular chromosome of a diploid cell. Combining these two criteria results in four possibilities. The combination of an artificial detector and alteration of gene expression have been tried several times: with strong overexpression from the GAL1 promoter (Duffy et al. 2016; Ouspenski et al. 1999), mild underexpression from hemizygous loci, and mild overexpression conferred by centromeric plasmids with genes under own promoters (Zhu et al. 2015). Two other combinations involved deletion strains (complete loss of gene function) and either a chromosomal fragment (Yuen et al. 2007) or intact natural chromosomes (Andersen et al. 2008; Choy et al. 2013; Kanellis et al. 2007; Yuen et al. 2007). The lists of genes identified in particular studies tended to overlap substantially and all were clearly enriched in functional categories known to be important for the stability of genomes. On the other hand, each of those studies pointed to a number of new genes as factors affecting genetic stability in yeast. One reason why results differ between screens is that studies relying on additional fragments of chromosomes tend to perform well in detecting chromosome loss, while those relying on natural chromosomes are better suited to reveal recombination (Acuña et al. 1994; Andersen et al. 2008; Yuen et al. 2007). Another is that an absence of a protein is likely to disturb the cell functioning in a different way than its excess (Oberdorf and Kortemme 2009; Veitia et al. 2013). In sum, the already used approaches to screening for the CIN genes provided both new hits and reassuring cross validations. However, it is still likely that a non-trivial number of potentially important genes has not been yet identified (Duffy et al. 2016; Stirling et al. 2011).

We set to expand systematic studies of CIN by introducing the lacking fourth combination: overexpression of single genes with $\mathrm{LOH}$ at natural chromosomes as detectors of instability. In our case, overexpression was very strong, because we used multi-copy plasmids with genes fused to the GAL1 promoter. This could result in intensification of regular functions of genes, but not only. Possible outcomes include excessive sequestration of partners, competition for subunits shared with other proteins, disruption of stoichiometric complexes, promiscuous interactions with proteins or nucleic acids, toxicity of misfolded aggregates, and others (Albrecht et al. 2018; Moriya 2015; Prelich 2012). Thus, overexpression studies can lead to unexpected and interesting results by creating perturbations which are rather artificial but, nevertheless, revealing.

We performed a genetic screen for increased rate of $\mathrm{LOH}$ in $S$. cerevisiae. We used a complete genomic collection of $2 \mu$ plasmids each carrying a single yeast gene under strong inducible promoter (Gelperin et al. 2005). LOH was marked by mutation to canavanine resistance which occurred when a functional allele was lost at a heterozygous $C A N 1 /$ canl locus residing on the chromosome $\mathrm{V}$ of the host cell (mutations inactivating CANl are much rarer). A co-occurring $\mathrm{LOH}$ at MET6/met6 on the opposite arm of the same chromosome signaled that an entire chromosome $\mathrm{V}$ was lost. The canavanine-resistance assay was quantitative, it allowed to estimate how much the frequency of LOH increased, and how often, it was caused by the loss of an entire chromosome. We provide a list of genes which caused a substantial, and often dramatic, increase in $\mathrm{LOH}$ upon strong overexpression. We also show that chromosomal instability depended on the identity and not amount of overexpressed protein even if the latter was enhanced by orders of magnitude.

\section{Materials and methods}

\section{Strains and plasmids}

We used a previously constructed collection of single yeast open-reading frames (ORFs), each with an inducible promoter $\mathrm{P}_{G A L I}$, cloned into a multi-copy 2-micron plasmid with the URA3 marker and hosted by the haploid yeast strain Y258 MATa pep4-3 his4-580 ura3-52 leu2-3. This so-called MORF collection is used to express proteins with a C-terminus fused affinity tag $\mathrm{His}_{6}$-HA-ZZ. As much as $93 \%$ of 5188 such constructs could be recovered from cell lysates and most of those appeared also to be intact in vivo (Gelperin et al. 2005). The host stain is derived from the S288c background (http://dharmacon.gelifesciences.com/resources/ faqs/y258-used-yeast-orf-collection-derived-s288c).

Figure 1 summarizes methods used to derive and assay our experimental strains. As the intention of the study was to test for stability of chromosomes in a mitotically dividing diploid cell, we had to turn the entire MORF collection of haploids into diploids. We first cured one of the haploid strains of its MORF plasmid and transformed it with another plasmid, $\mathrm{p} U R A 3 \mathrm{P}_{G A L I}-H O$. After transient induction of the $H O$ gene with galactose, a diploid strain was obtained, cured of the plasmid, sporulated, and dissected to derive an $M A T \underline{\alpha}$ haploid strain. This new strain was a stable haploid and fully isogenic to the original Y258, except for the mating type. Two further changes were introduced into it. First, we exposed it to $60 \mathrm{mg} / \mathrm{l}$ canavanine to select for $\mathrm{Can}^{\mathrm{R}}$ mutants. One of them, confirmed to have a frame-shift mutation in $C A N 1$, was selected for the next step, that is, replacing the MET6 gene with a cassette providing resistance to geneticin, yielding met6::kanMX4. (CAN1 and MET6 reside, respectively, on the left and right arms of chromosome V.)

The obtained MAT $\underline{\alpha}$ strain was used as a single mating partner for all MATa plasmid-hosting strains. The mating was done by mixing aliquots of $10 \mu \mathrm{l}$ of the MAT $\underline{\alpha}$ strain with the same volume of a single MORF strain in 96-well 
titration plates and adding $180 \mu \mathrm{l}$ of fresh YPD. Diploids were selected through three consecutive transfers into synthetic minimal glucose (SD) medium supplemented with leucine, histidine, and $200 \mathrm{mg} / \mathrm{l}$ geneticin. The resultant collection of the overexpression plasmids in double heterozygous diploid cells, CAN1/canl HIS6/his6::kanMX4, was stored at $-70{ }^{\circ} \mathrm{C}$. To keep the $\mathrm{P}_{G A L I}$ promoter repressed, the only carbon source used when handling both the haploid and diploid collections was glucose.

\section{Screen for LOH with canavanine-resistance assay}

Frozen samples of the diploid collection were thawed and transferred with a 96-pin replicator onto SD agar supplemented with leucine, histidine, and geneticin. In this and all other experiments, the temperature of incubation was kept at $30^{\circ} \mathrm{C}$. The $\mathrm{P}_{G A L I}$ promoter is turned on in two steps. First, its repressor (glucose) is removed, and this is done using raffinose as carbon source. Then, galactose acting as an activator is added (Gelperin et al. 2005). In our experiment, after initial micro-cultures reached the stationary phase density in glucose, samples of $2 \mu \mathrm{l}$ were transferred to $198 \mu \mathrm{l}$ of the same glucose-based medium. The second transfer to glucose was done to synchronize the growth phase of the microcultures. After $48 \mathrm{~h}$ of incubation, $20 \mu \mathrm{l}$ samples were transferred to $180 \mu \mathrm{l}$ of synthetic complete (SC) medium lacking uracil and with $2 \%$ raffinose replacing glucose. After $72 \mathrm{~h}$ of incubation, $5 \mu \mathrm{l}$ samples were transferred to $195 \mu \mathrm{l}$ of new medium, the same as above plus $2 \%$ galactose (Raffinose was required as Y258 does not utilize galactose as a source of energy). After $48 \mathrm{~h}$ of incubation, $4 \mu \mathrm{l}$ samples were put onto agar SC medium (96 per one rectangular plate) which had $2 \%$ glucose as the sole source of carbon, lacked arginine and was supplemented with $60 \mathrm{mg} / \mathrm{l}$ canavanine. The agar plates were photographed after $48 \mathrm{~h}$ and then kept at $4{ }^{\circ} \mathrm{C}$. Enlarged photographs and the original plates were evaluated independently by two experimenters looking for visible enrichment in the number of $\mathrm{Can}^{\mathrm{R}}$ mutants (identity of the evaluated strains was unknown during this inspection). The criteria used to decide about enhanced $\mathrm{LOH}$ are described in "Results".

\section{Test for chromosome loss with methionine auxotrophy assay}

This experiment was done in the same way as the $\mathrm{LOH}$ screen except for the final transfer of samples of galactoseinduced cultures onto agar with canavanine. These samples were placed not as 96-spot arrays but on individual $90 \mathrm{~mm}$ dishes. The volume of individual samples varied, and the goal was to obtain about 200 colonies of $\mathrm{Can}^{\mathrm{R}}$ mutants per plate which then could be replica plated onto SD agar supplemented with leucine and histidine but not methionine.
At least three plates per every strain classified as one with enhanced LOH were replica plated.

\section{Other methods}

To measure the level of protein overexpression, the studied strains were transferred through media based on glucose, raffinose, and raffinose with galactose in the way described above. In the last medium, cells were harvested after $24 \mathrm{~h}$ of incubation, washed with ice cold water and frozen. To start protein extraction, the cells were beaten with glass beads in $100 \mu \mathrm{l}$ of lysis buffer $(50 \mathrm{mM}$ Tris-HCl, $\mathrm{pH} 7.5,0.5 \%$ SDS, $0.1 \mathrm{mM}$ EDTA, protease inhibitors) for $4 \mathrm{~h}$ in $4{ }^{\circ} \mathrm{C}$. Afterwards, cell remnants were spun down and supernatant was collected. Total protein content was determined using a BCA protocol. The same supernatant was used for a competitive ELISA assay aimed at determining the cellular level of the overexpressed proteins (Tomala and Korona 2013).

The cellular content of reactive oxygen species was determined with CellROX ${ }^{\circledR}$ Deep Red dye (Life Technologies). Cells were grown and harvested in the same way as in the protein assay. The dye was added at the working concentration of $5 \mu \mathrm{M}$. After the dying, samples of cells were washed in PBS and lysed with lithium acetate and SDS. After spinning down the cell debris, supernatants of the resulting samples were measured for fluorescence with $635 \mathrm{~nm}$ of the excitation and $665 \mathrm{~nm}$ of emission wavelength. The use of cell extracts, instead of intact cells, has been found more reliable for this particular dying kit (James et al. 2015).

\section{Results}

\section{Can $^{R}$ screen for increased LOH}

We screened a set of 5179 diploid strains carrying single overexpression plasmids, arrayed individually on 96-well titration plates. We grew them on medium containing galactose to induce overexpression and then transferred samples onto agar plates with canavanine (and glucose to stop overexpression). The size of samples was adjusted to yield 5-10 $\mathrm{Can}^{\mathrm{R}}$ colonies as the most frequent result. We found 636 candidates for enhanced LOH by applying an intentionally liberal criterion: the number of colonies had to be at least three times higher. Supplementary Table S1 lists these genes. This set was likely to include a fair number of strains with "standard" rate of LOH but ones in which an enhanced number of mutants occurred by chance. To narrow the search, all 636 candidate strains were re-assayed in two independent runs. This time, densities of $\mathrm{Can}^{\mathrm{R}}$ colonies per patch were classified into three ranks of increasing LOH. Figure 1 illustrates how the patches of colonies were divided into three ranks: standard, dense, and very dense, that is, filled with 

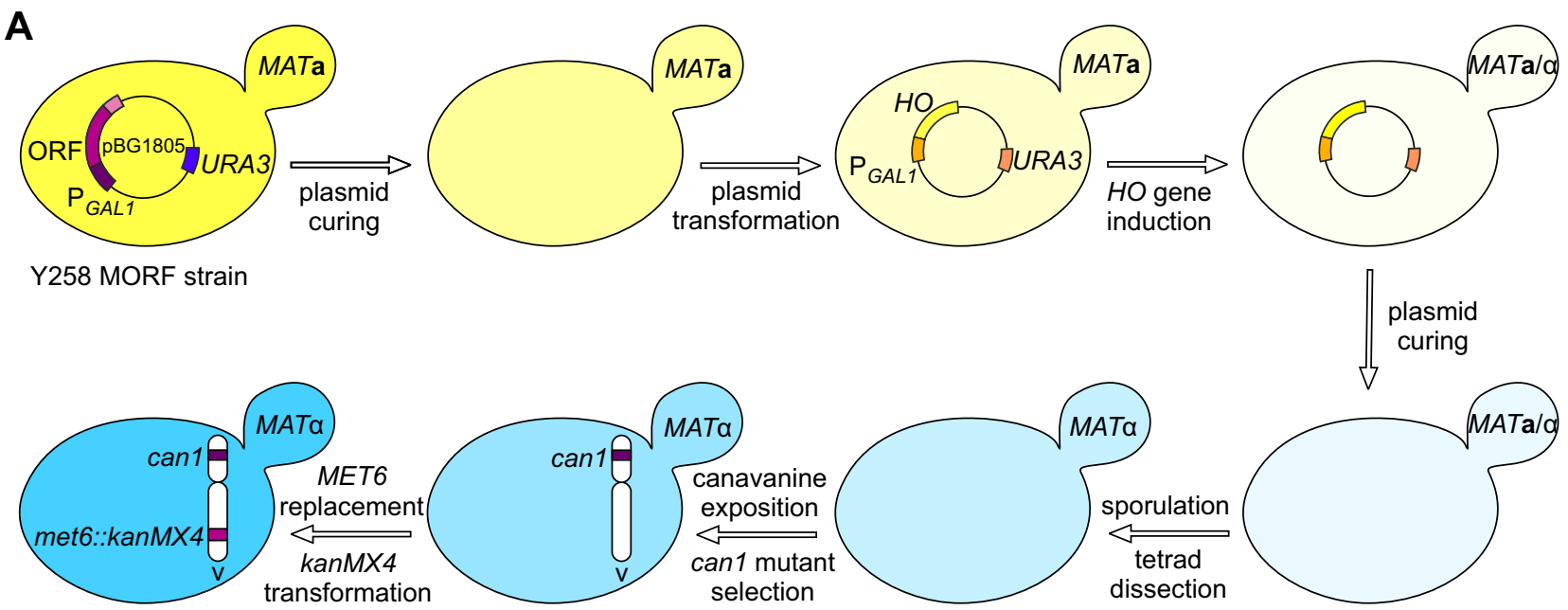

universal mater
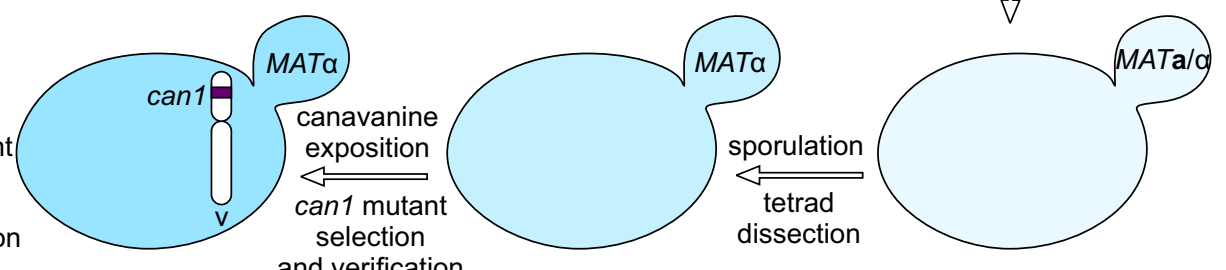

B

MATa MORF collection

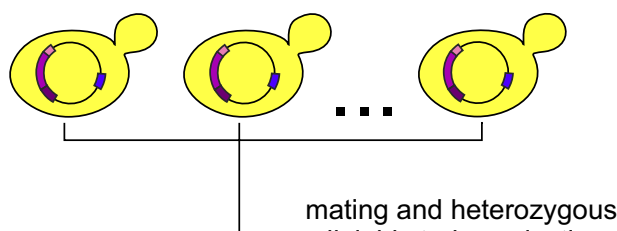
diploid strains selection

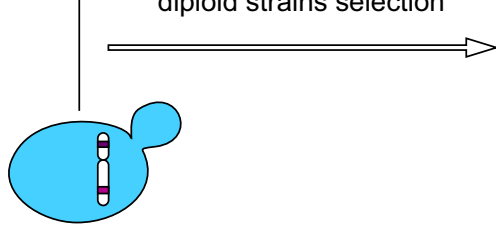

universal mater
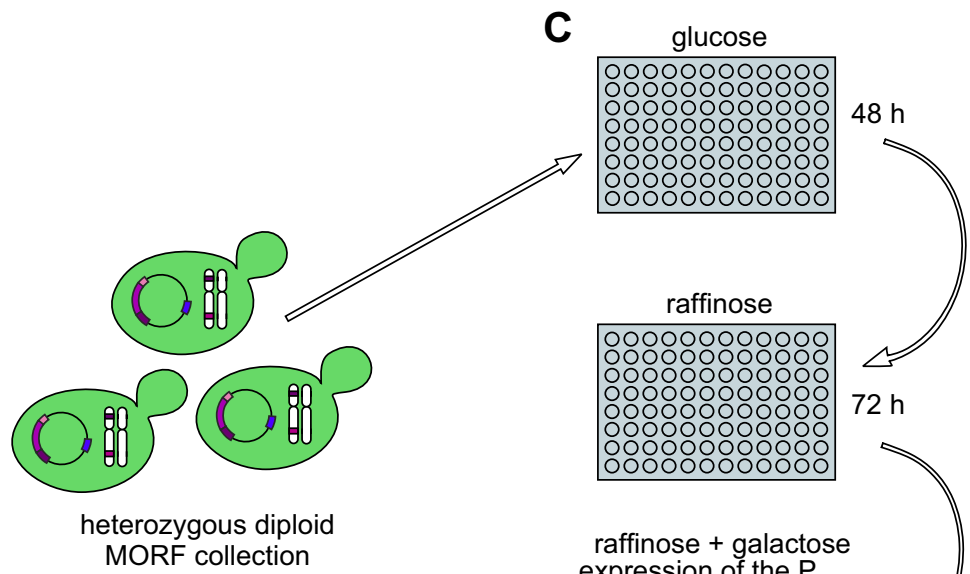

$72 \mathrm{~h}$

raffinose + galactose expression of the $\mathrm{P}_{G A L}$ MORF collection

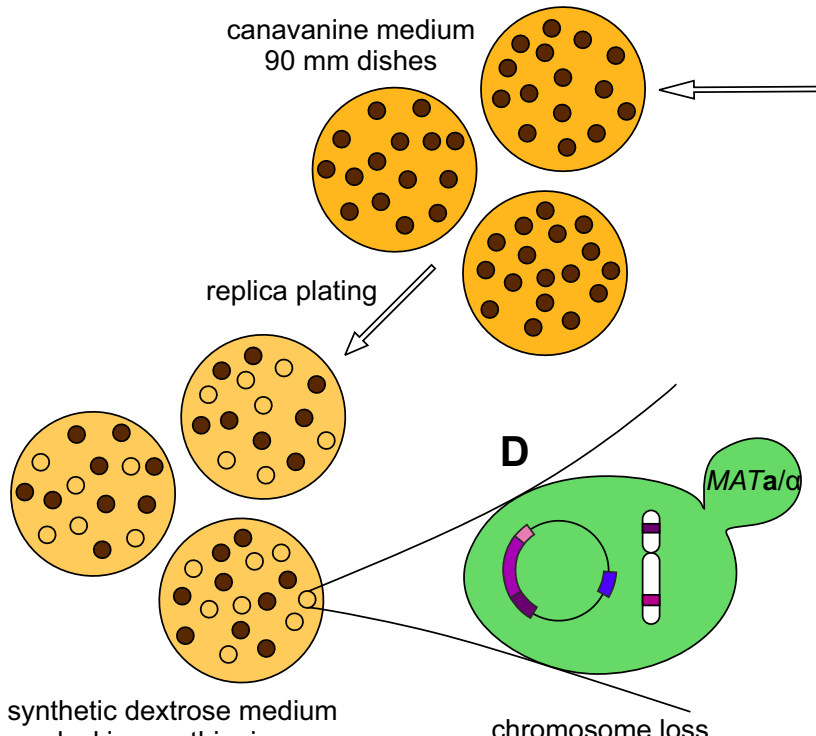
test for chromosome loss

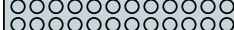

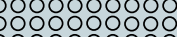
O०

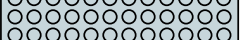
(selected strains)

$$
\sqrt{\nabla}
$$

screen for $\mathrm{LOH}$ canavanine medium plate

\begin{tabular}{|c|c|c|c|c|c|c|c|c|c|c|c|}
\hline \$ & & $\therefore$ & .6 & 2 & 8 & 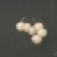 & $\sigma^{\circ}$ & & 25 & & \\
\hline$\therefore$ & $\because$ & $\therefore$ & • & $:$ & 28 & : & : & $\therefore$ & : & • & •. \\
\hline • & 2 & $:$ & (1) & $\ldots$ & & $\bullet$ & s8: & (2) & ఈे & $\therefore$ & • \\
\hline i: & $\vdots$ & . & & $\therefore$ & & $y$ & 3 & is & $:$ & • & (1) \\
\hline & 4 & • & $:$ & & & • & $8:$ & : & • & (1) & $\because$ \\
\hline & • & $:$ & & & • & the & 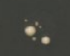 & $\because$ & تب. & (1) & : \\
\hline & & : & $\%$ & (1) & $\cdots$ & $\therefore$ & $40^{\circ}$ & A & • & (1) & : \\
\hline & $\approx$ & $\mathbf{s}^{3}$ & 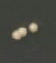 & 2 & & - & $\bullet^{\bullet}$ & $\bullet$ & - & & 2 \\
\hline
\end{tabular}




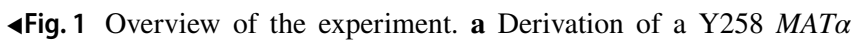
strain. The final construct was strictly isogenic to the original MATa strain (host of the whole collection) except for the presence of two markers introduced to chromosome $\mathrm{V}$ and absence of a MORF plasmid. b MAT $\alpha$ strain was then used as a universal mater to create a collection of heterozygous strains bearing MORF plasmids with single yeast genes under an inducible promoter, $\mathrm{P}_{G A L I}$. c Test for enhanced $\mathrm{LOH}$. Cultures were gradually induced to high expression of genes residing on the MORF plasmid. Aliquots of $5 \mu \mathrm{l}$ were exposed to canavanine medium yielding highly variable numbers of $\mathrm{Can}^{\mathrm{R}}$ colonies. A group of 636 candidate strains selected after an initial whole-genome screen was tested in two additional trials and ranked as very dense (1), dense (2) and standard (no labels). Those which exhibited consistently enhanced LOH in further tests (see the main text) were assayed for the frequency of chromosome $\mathrm{V}$ loss which was marked by an inability to grow on media-lacking methionine (d)

colonies. Ranks of the two replicas generally did correlate with each other (Pearson's $r=0.501 ; t=14.576, d f=634$, $p<<0.0001)$. However, two replicas of a single strains often differed considerably. It was understandable given the random nature of mutational events, but it also undermined reliability of any decision about the impact of individual genes on LOH. Therefore, we grouped the 636 strains into GO (Gene Ontology) functional categories. We then calculated a mean rank of patch density for every category (for those with more than 4 members among the 636 candidates). The category ranks were compared with the mean rank of a control group of 200 strains (randomly chosen from among the non-candidate strains). We found a statistically significant difference for 11 categories (partially overlapping) with 97 unique genes (Supplementary Table S2).

\section{Genes enhancing LOH}

The 97 genes were subjected to further tests to confirm that they indeed increased $\mathrm{LOH}$ and find out how often $\mathrm{LOH}$ resulted from the loss of an entire chromosome. For each tested strain, replicate samples of size sufficiently large to contain hundreds of $\mathrm{Can}^{\mathrm{R}}$ mutants were used. After the $\mathrm{Can}^{\mathrm{R}}$ colonies were counted, they were replica plated to test for the $\mathrm{Met}^{-}$phenotype marking LOH at the MET6 locus. Figure 2 shows 39 (out of 97) strains in which the mean frequency of $\mathrm{LOH}$ at the CANI locus was confirmed to be higher (at least two times) than in the control strains (control was composed of 28 randomly chosen strains which showed about median $\mathrm{LOH}$ in former tests). Both in the control and the confirmed 39 strains, $\mathrm{LOH}$ at $C A N 1$ was frequently accompanied by $\mathrm{LOH}$ at MET6. We assumed that a double LOH indicated the loss of an entire chromosome V. Not only because double recombination, double mutation or simultaneous recombination at one marker and mutation at the other are less likely to occur than a loss of a chromosome. We also observed that the $\mathrm{Can}^{\mathrm{R}}$ $\mathrm{Met}^{-}$colonies were distinctly smaller than the $\mathrm{Can}^{\mathrm{R}} \mathrm{Met}^{+}$ ones indicating growth disadvantage typical for monosomics (Alvaro et al. 2006).

Regarding functions of the 39 genes, 32 of them relate to cell division. Most of them are known to be needed at different stages of mitosis beginning with establishment of cell polarity until the late cell division checkpoints. Only few $-C D C 7$, $H O P 1, M S C 6, R C K 1$, and REC8 - are thought to participate in meiosis, and therefore, it might have been destructive to express them under vegetative growth.

The seven remaining genes were categorized by a Gene Ontology analysis tool as involved in "metabolism of sulfur compounds" (Eden et al. 2009b). They have no clear links to cell division, but could possibly destabilize functioning of the cell, including cell division, if overabundance of their products resulted in an excessive level of reactive oxygen species (ROS). $L S C 2$, which upon overexpression destabilized the genome more than any other gene, appears to also excite ROS to the highest level (Fig. 3).

\section{Frequent loss of an entire chromosome in the presence of galactose}

The median frequency of $\mathrm{Can}^{\mathrm{R}}$ mutants in the control was $1.1 \times 10^{-4}$ which is about average for $\mathrm{LOH}$ in a mitotically dividing diploid yeast cell (Andersen et al. 2008). More intriguing was the observed high frequency of double $\mathrm{LOH}$, because in S. cerevisiae the combined frequency of events involving chromosome recombination, breakage, or repair is typically higher than the loss of an entire chromosome (Andersen et al. 2008; McMurray and Gottschling 2003).

We hypothesized that the observed high frequency of the loss of chromosome could depend on the specific medium we used. We repeated the tests of $\mathrm{LOH}$ for the control strains with the medium containing raffinose and galactose but also with media containing only glucose or only raffinose. Figure $4 \mathrm{a}$ shows that simultaneous $\mathrm{LOH}$ at both loci was less frequent than single $\mathrm{LOH}$ at $C A N 1$ when glucose or raffinose was used. Thus, the strain used by us was not specific, or defective, in a way that would make the loss of an entire chromosome especially frequent. Only for raffinose plus galactose, when the $\mathrm{P}_{G A L I}$ promoter was fully active, the loss of an entire chromosome prevailed over other events underpinning $\mathrm{LOH}$. Figure $4 \mathrm{~b}$ demonstrates that galactose is a stimulator of $\mathrm{LOH}$ only when an MORF plasmid is present. This result provides no support for an alternative hypothesis stating that galactose may be a metabolic agent disturbing the cell division regardless of its interaction with the target promoter.

\section{Rate of LOH was determined by the function, not amount of overexpressed protein}

The host strain, Y258, lacks the PEP4 gene coding for proteinase $\mathrm{A}$ and, therefore, tends to accumulate peptides and 


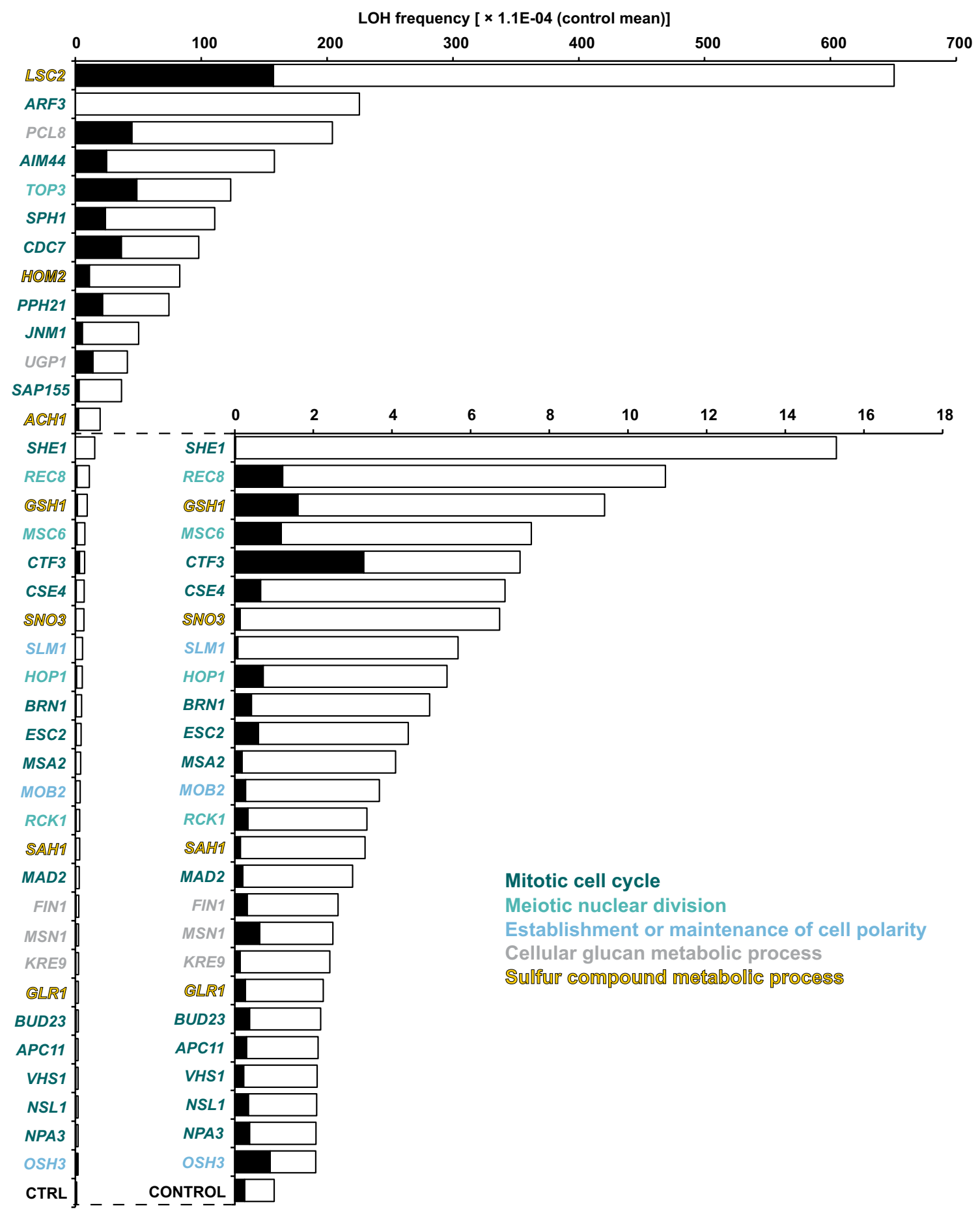

Fig. 2 Genes which increase frequency of LOH more than twice when overexpressed. Frequency of $\mathrm{LOH}$ at the CAN1/canl locus is represented by filled bars. $\mathrm{LOH}$ at both $C A N 1 /$ canl and MET6/met6 (loss of chromosome V) is represented by empty bars. Control con-

proteins, including those with abnormal structures (Parr et al. 2007). We hypothesized that a very high overexpression of a single protein, irrespective of its function, could be a large enough burden for the cell to disturb mitosis.

sisted of 28 strains randomly selected from among those in which $\mathrm{LOH}$ was observed with about median frequency (see the main text). Average frequency of total $\mathrm{LOH}$ in the control 28 strains was $1.01 \times 10^{-4}$ and is used as a unit to scale the horizontal axis

Figure 5 shows that this was not the case. Although the cellular content of proteins varied widely, there was no correlation between the amount of an expressed protein and the rate of $\mathrm{LOH}$. 


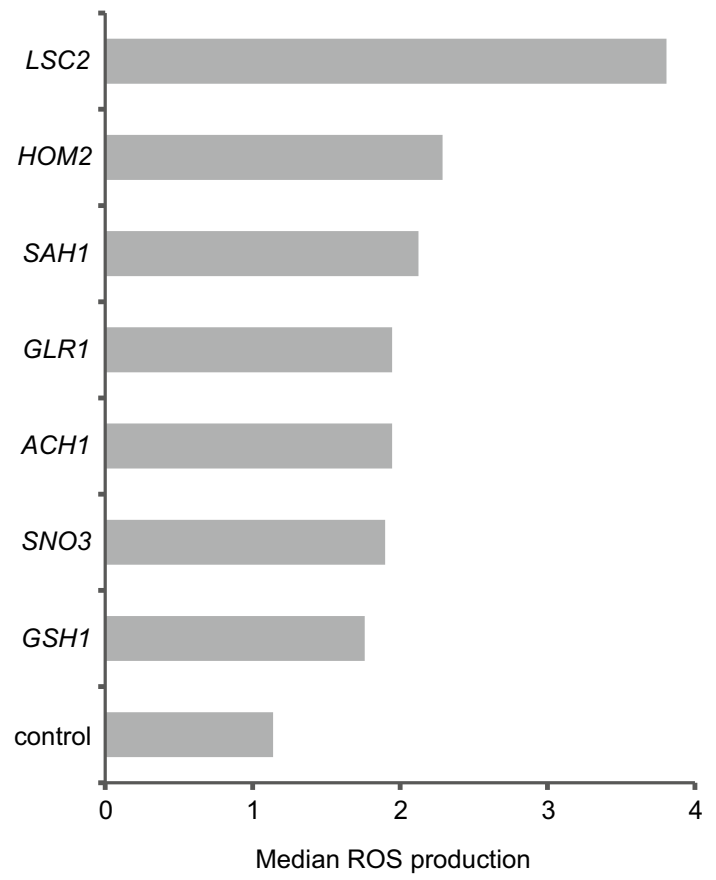

Fig. 3 Cellular level of ROS after overexpression. The seven genes belonging to the category of metabolism of sulfur compounds are compared with a control obtained as a mean of three randomly chosen genes judged "standard" (see Fig. 1)

\section{Discussion}

Stable replication and segregation of chromosomes requires concerted work of hundreds of proteins. It is difficult to predict a priori which of them are critically

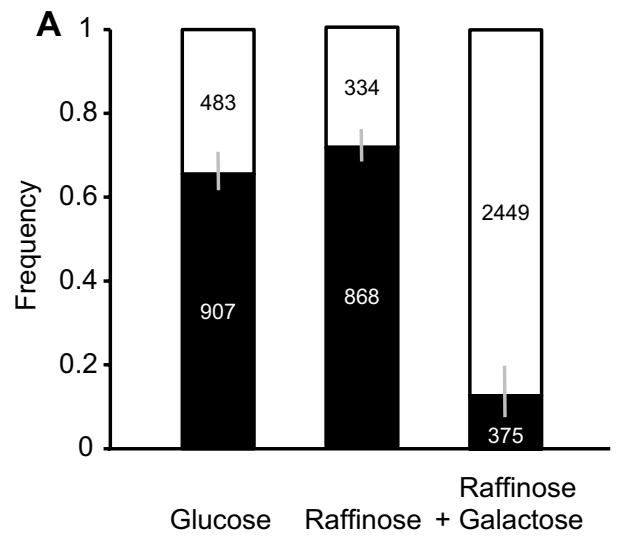

Fig. 4 a Relative frequencies of the two types of $\mathrm{LOH}$ in the control strains: single $\mathrm{LOH}$ at the CAN1/canl locus (black field) and double $\mathrm{LOH}$ at both the CAN1/canl and MET6/met6 loci (open field) with SE shown as bars. Numbers of colonies used to calculate the frequencies are shown in relevant fields. Cultures were passed sequentially through the three media used to induce overexpression-SC with the source of organic carbon being glucose, raffinose or raffinose important and, even more so, which are not important for the stability of chromosomes. We used a collection of yeast diploid strains in which each single gene was abundantly overexpressed from a plasmid. The strains were heterozygous at the CANI locus of chromosome V. This simple, although not yet tried experimental setting, proved successful as we found 39 genes that increased LOH when overexpressed. Of those, 22 are new for the CIN research (Supplementary Table S3).

Among the 39 genes increasing LOH upon overexpression, as many as 28 were within GO categories obviously linked to cell polarity and progression of cell cycle. Of those, as many as 13 related to sister chromatid segregation. There were also genes known to function primarily in meiosis and, therefore, possibly interfering with mitosis when expressed untimely. On top of the 28 genes directly linked to the cell cycle, there were four genes-KRE8, MSN1, PCL8, and $U G P 1$ - known as primarily involved in glucan metabolism. $\beta$-glucan is an essential component of the yeast cell wall, making more than half of its dry weight (Lesage and Bussey 2006). It is also a major component of the septum which forms between the mother and daughter cells (Roncero and Sánchez 2010). Disturbed metabolism of glucan results in improper development of a bud and septum which arrests the cell cycle prior to spindle body separation (Suzuki et al. 2004). This phenomenon has been called the cell wall integrity checkpoint (Negishi and Ohya 2010). Adding these four genes, as many as 32 out of the total of 39 genes increasing $\mathrm{LOH}$ can be related to cell cycle progression. This is hardly surprising. However, the paucity of genes directly involved in DNA metabolism is remarkable. Only one of the 39 gene products, the Top3 topoisomerase, is an enzyme for which

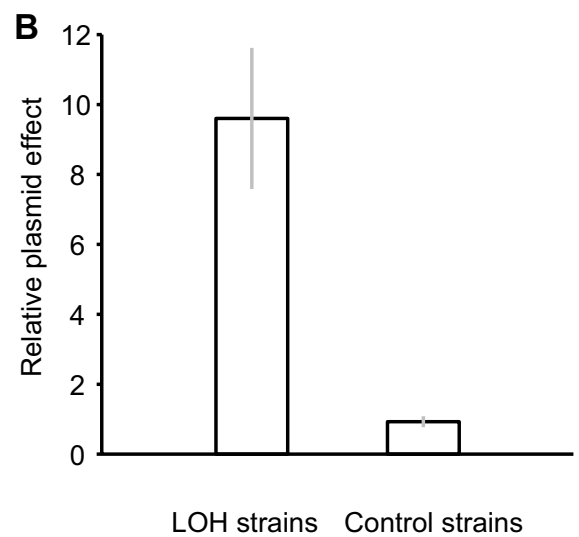

with galactose-with $\mathrm{LOH}$ being scored at the end of each phase of growth. b Effect of a plasmid on the frequency of LOH. The tested 39 strains and control 28 strains (compare Fig. 2) were cured of plasmid. The ratio of the frequency of $\mathrm{LOH}$ with and without plasmid was calculated for each strain and averaged for the two groups. Bars show standard errors 


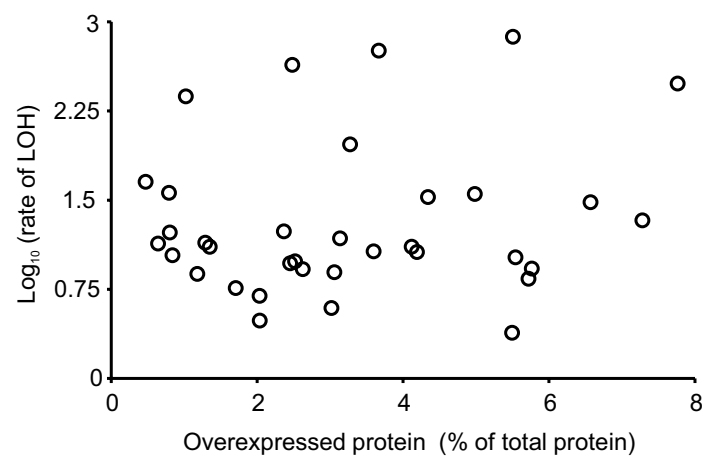

Fig. 5 Relation between the amount of overproduced protein (as a percent of total cellular protein) and the rate of LOH. Pearson's correlation coefficient was not statistically significant: $r=0.160, d f=33$, $p=0.718$. Four of the 39 genes shown in Fig. 2-FIN1, MOB2, $O S H 3$, and SLM1-were not included in this analysis, because the corresponding estimates of protein content were lost accidentally

DNA is a substrate. In former screens, much larger fraction of genes promoting $\mathrm{LOH}$ was linked to synthesis, recombination, transcription, or repair of DNA. This was especially true for gene deletions (Andersen et al. 2008; Eden et al. 2009a; Mikolaskova et al. 2018; Stirling et al. 2011). Our results suggest that overproduction of a single protein can be damaging to the coordination of complex processes necessary to complete cell division. In the case of DNA synthesis and repair, it is an absence rather than overabundance of a single protein that strongly interferes with the process of timely replication and separation of DNA strands. However, it is worth remembering that we used a collection of fusion proteins under truly heavy overexpression. It could be that not the core proteins, but their added components were more disturbing to some functions (cell division) than to others (DNA repair). In addition, overexpression is typically pleiotropic and generally proteotoxic. Therefore, caution is needed when making functional generalizations about the observed CIN patterns.

The seven remaining genes participate in the metabolism of sulfur compounds (among them, SNO3 is only indirectly linked to this activity, and its specific role is still unknown). However, individual roles of these genes are different, even contradictory. For example, GSHI and GLRI code for a synthetase and reductase of glutathione, respectively. Glutathione is a major scavenger of reactive oxygen species, ROS (Deponte 2013), but it can impact, often indirectly, a variety of other cellular processes, including nuclear division (Fidai et al. 2016; Kaniak-Golik and Skoneczna 2015; Kumar et al. 2011; Matsuo et al. 2017). Deciphering the exact roles of these genes in maintaining genome stability would require work much broader than presented in this paper. We note, however, that the very strong destabilizing effect of Lsc2 observed here may have a straightforward explanation. Overabundance of this protein should result in an excessive level of succinate released from succinyloCoA (Fleck and Brock 2009; Przybyla-Zawislak et al. 1998). Succinate is a source of electrons for the Complex II of the electron transport chain. Electrons escaping an excessively active transport chain form ROS which can be severely damaging to macromolecules, including DNA (Herrero et al. 2008).

Another result of our study is the observation that the proportion of whole-chromosome loss can vary substantially depending on medium. The strain used here hosted multiple copies of a 2-micron plasmid with a single yeast ORF fused to the $P_{G A L I}$ promoter. One can safely assume that the number of plasmid copies per cell was high in glucose and raffinose and decreased after overexpression was turned on by galactose. This is, because the overexpressed protein is a burden, and therefore, cells with low numbers of plasmids are selected for (Makanae et al. 2013). Thus, not the very presence of abundant plasmid DNA but rather an intense transcription of its DNA was responsible for the observed high frequency of chromosome loss. Frequent unwinding and separation of DNA strands at the plasmid might have led to more intense interactions with chromosomes resulting in improper segregation of the latter. Indeed, it has been found that the 2-micron plasmid and chromosomes interact with each other. Specifically, the plasmid codes for a plasmid-chromosome recombination system which couples segregating chromosomes with plasmids to prevent both extinction and runaway proliferation of the latter (Mehta et al. 2002; Rizvi et al. 2017; Yen-Ting-Liu et al. 2014).

Several recent reviews attempted to list yeast genes which potentially affect the stability of chromosomes when their expression is absent or set at a non-native level (Choy et al. 2013; Duffy et al. 2016; Eden et al. 2009a; Stirling et al. 2011; Zhu et al. 2015). There are hundreds of such genes and the already long lists may not yet be complete. The stability of chromosomes is obviously a truly polygenic trait. Future research should probably concentrate on understanding which genes are important universally and which become relevant only under specific conditions.

Acknowledgements Funds were provided by grants from Polish National Science Centre, nos. 2013/11/B/NZ2/00122 and 2017/25/B/ NZ2/01036.

Open Access This article is distributed under the terms of the Creative Commons Attribution 4.0 International License (http://creativeco mmons.org/licenses/by/4.0/), which permits unrestricted use, distribution, and reproduction in any medium, provided you give appropriate credit to the original author(s) and the source, provide a link to the Creative Commons license, and indicate if changes were made. 


\section{References}

Acuña G, Würgler FE, Sengstag C (1994) Reciprocal mitotic recombination is the predominant mechanism for the loss of a heterozygous gene in Saccharomyces cerevisiae. Environ Mol Mutagen. https://doi.org/10.1002/em.2850240408

Albrecht D, Hürlimann HC, Ceschin J, Saint-Marc C, Pinson B, Daignan-Fornier B (2018) Multiple chemo-genetic interactions between a toxic metabolite and the ubiquitin pathway in yeast. Curr Genet 1-12

Alvaro D, Sunjevaric I, Reid RJ, Lisby M, Stillman DJ, Rothstein R (2006) Systematic hybrid LOH: a new method to reduce false positives and negatives during screening of yeast gene deletion libraries. Yeast 23:1097-1106

Andersen MP, Nelson ZW, Hetrick ED, Gottschling DE (2008) A genetic screen for increased loss of heterozygosity in Saccharomyces cerevisiae. Genetics 179:1179-1195

Boone C (2014) Yeast systems biology: our best shot at modeling a cell. Genetics 198:435-437

Choy JS, O'Toole E, Schuster BM, Crisp MJ, Karpova TS, McNally JG, Winey M, Gardner MK, Basrai MA (2013) Genome-wide haploinsufficiency screen reveals a novel role for $\gamma$-TuSC in spindle organization and genome stability. Mol Biol Cell 24:2753-2763

Comai L (2005) The advantages and disadvantages of being polyploid. Nat Rev Genet 6:836-846

Deponte M (2013) Glutathione catalysis and the reaction mechanisms of glutathione-dependent enzymes. Biochim Biophys Acta (BBA) Gen Subj 1830:3217-3266

Duffy S, Fam HK, Wang YK, Styles EB, Kim J-H, Ang JS, Singh T, Larionov V, Shah SP, Andrews B (2016) Overexpression screens identify conserved dosage chromosome instability genes in yeast and human cancer. Proc Natl Acad Sci 113:9967-9976

Duijf P, Benezra R (2013) The cancer biology of whole-chromosome instability. Oncogene 32:4727-4736

Dunham MJ, Fowler DM (2013) Contemporary, yeast-based approaches to understanding human genetic variation. Curr Opin Genet Dev 23:658-664

Eden E, Navon R, Steinfeld I, Lipson D, Yakhini Z (2009a) GOrilla: a tool for discovery and visualization of enriched GO terms in ranked gene lists. BMC Bioinform 10:48

Eden E, Navon R, Steinfeld I, Lipson D, Yakhini Z (2009b) GOrilla: a tool for discovery and visualization of enriched GO terms in ranked gene lists. BMC Bioinform 10:1

Fidai I, Wachnowsky C, Cowan J (2016) Glutathione-complexed [2Fe2S] clusters function in Fe-S cluster storage and trafficking. J Biol Inorg Chem 21:887-901

Fleck CB, Brock M (2009) Re-characterisation of Saccharomyces cerevisiae Ach1p: fungal CoA-transferases are involved in acetic acid detoxification. Fungal Genet Biol 46:473-485

Gelperin DM, White MA, Wilkinson ML, Kon Y, Kung LA, Wise KJ, Lopez-Hoyo N, Jiang L, Piccirillo S, Yu H (2005) Biochemical and genetic analysis of the yeast proteome with a movable ORF collection. Genes Dev 19:2816-2826

Giaever G, Nislow C (2014) The yeast deletion collection: a decade of functional genomics. Genetics 197:451-465

Harari Y, Ram Y, Kupiec M (2018) Frequent ploidy changes in growing yeast cultures. Curr Genet 64(5):1001-1004

Herrero E, Ros J, Bellí G, Cabiscol E (2008) Redox control and oxidative stress in yeast cells. Biochim Biophys Acta (BBA) Gen Subj 1780:1217-1235

Hui L, Bianchi DW (2013) Recent advances in the prenatal interrogation of the human fetal genome. Trends Genet 29:84-91

James J, Fiji N, Roy D, Shihabudeen MS, Chattopadhyay D, Thirumurugan $\mathrm{K}$ (2015) A rapid method to assess reactive oxygen species in yeast using H 2 DCF-DA. Anal Methods 7:8572-8575
Kanellis P, Gagliardi M, Banath JP, Szilard RK, Nakada S, Galicia S, Sweeney FD, Cabelof DC, Olive PL, Durocher D (2007) A screen for suppressors of gross chromosomal rearrangements identifies a conserved role for PLP in preventing DNA lesions. PLoS Genet 3:e134

Kaniak-Golik A, Skoneczna A (2015) Mitochondria-nucleus network for genome stability. Free Radic Biol Med 82:73-104

Kumar C, Igbaria A, D'autreaux B, Planson AG, Junot C, Godat E, Bachhawat AK, Delaunay-Moisan A, Toledano MB (2011) Glutathione revisited: a vital function in iron metabolism and ancillary role in thiol-redox control. EMBO J 30:2044-2056

Lesage G, Bussey H (2006) Cell wall assembly in Saccharomyces cerevisiae. Microbiol Mol Biol Rev 70:317-343

Makanae K, Kintaka R, Makino T, Kitano H, Moriya H (2013) Identification of dosage-sensitive genes in Saccharomyces cerevisiae using the genetic tug-of-war method. Genome Res 23:300-311

Matsuo R, Mizobuchi S, Nakashima M, Miki K, Ayusawa D, Fujii M (2017) Central roles of iron in the regulation of oxidative stress in the yeast Saccharomyces cerevisiae. Curr Genet 63:895-907

McMurray MA, Gottschling DE (2003) An age-induced switch to a hyper-recombinational state. Science 301:1908-1911

Mehta S, Yang XM, Chan CS, Dobson MJ, Jayaram M, Velmurugan S (2002) The 2 micron plasmid purloins the yeast cohesin complex. J Cell Biol 158:625-637

Mikolaskova B, Jurcik M, Cipakova I, Kretova M, Chovanec M, Cipak L (2018) Maintenance of genome stability: the unifying role of interconnections between the DNA damage response and RNA-processing pathways. Curr Genet 64(5):971-983

Moriya H (2015) Quantitative nature of overexpression experiments. Mol Biol Cell 26:3932-3939

Negishi T, Ohya Y (2010) The cell wall integrity checkpoint: coordination between cell wall synthesis and the cell cycle. Yeast 27:513-519

Oberdorf R, Kortemme T (2009) Complex topology rather than complex membership is a determinant of protein dosage sensitivity. Mol Syst Biol 5:253

Otto SP, Whitton J (2000) Polyploid incidence and evolution. Annu Rev Genet 34:401-437

Ouspenski II, Elledge SJ, Brinkley B (1999) New yeast genes important for chromosome integrity and segregation identified by dosage effects on genome stability. Nucleic Acids Res 27:3001-3008

Parr CL, Keates RA, Bryksa BC, Ogawa M, Yada RY (2007) The structure and function of Saccharomyces cerevisiae proteinase A. Yeast 24:467-480

Prelich G (2012) Gene overexpression: uses, mechanisms, and interpretation. Genetics 190:841-854

Przybyla-Zawislak B, Dennis RA, Zakharkin SO, McCammon MT (1998) Genes of succinyl-CoA ligase from Saccharomyces cerevisiae. FEBS J 258:736-743

Rizvi SMA, Prajapati HK, Ghosh SK (2017) The 2 micron plasmid: a selfish genetic element with an optimized survival strategy within Saccharomyces cerevisiae. Curr Genet 64(1):25-42

Roncero C, Sánchez Y (2010) Cell separation and the maintenance of cell integrity during cytokinesis in yeast: the assembly of a septum. Yeast 27:521-530

Scherens B, Goffeau A (2004) The uses of genome-wide yeast mutant collections. Genome Biol 5:1

Sheltzer JM, Amon A (2011) The aneuploidy paradox: costs and benefits of an incorrect karyotype. Trends Genet 27:446-453

Stirling PC, Bloom MS, Solanki-Patil T, Smith S, Sipahimalani P, Li Z, Kofoed M, Ben-Aroya S, Myung K, Hieter P (2011) The complete spectrum of yeast chromosome instability genes identifies candidate CIN cancer genes and functional roles for ASTRA complex components. PLoS Genet 7:e1002057 
Suzuki M, Igarashi R, Sekiya M, Utsugi T, Morishita S, Yukawa M, Ohya Y (2004) Dynactin is involved in a checkpoint to monitor cell wall synthesis in Saccharomyces cerevisiae. Nat Cell Biol 6:861-871

Tomala K, Korona R (2013) Evaluating the fitness cost of protein expression in Saccharomyces cerevisiae. Genome Biol Evol 5:2051-2060

Torres EM, Williams BR, Amon A (2008) Aneuploidy: cells losing their balance. Genetics 179:737-746

Veitia RA, Bottani S, Birchler JA (2013) Gene dosage effects: nonlinearities, genetic interactions, and dosage compensation. Trends Genet 29:385-393
Yen-Ting-Liu SS, Ma C-H, Kachroo AH, Rowley PA, Chang K-M, Fan H-F, Jayaram M (2014) The partitioning and copy number control systems of the selfish yeast plasmid: an optimized molecular design for stable persistence in host cells. Microbiol Spectr. https ://doi.org/10.1128/microbiolspec.PLAS-0003-2013.

Yuen KW, Warren CD, Chen O, Kwok T, Hieter P, Spencer FA (2007) Systematic genome instability screens in yeast and their potential relevance to cancer. Proc Natl Acad Sci 104:3925-3930

Zhu J, Heinecke D, Mulla WA, Bradford WD, Rubinstein B, Box A, Haug JS, Li R (2015) Single-cell based quantitative assay of chromosome transmission fidelity. G3 Genes Genomes Genet 5:1043-1056 